\title{
Establishment of an in vivo rat model for chronic musculoskeletal implant infection
}

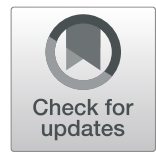

Eivind Wits $\varnothing^{* *}$, Linh Hoang ${ }^{2}$, Kirsti Løseth² and Kåre Bergh ${ }^{2,3}$

\begin{abstract}
Background: The aim of the study was to establish an experimental chronic musculoskeletal infection model in vivo characterized by (a) a small bacterial inoculum, (b) no general or local signs of infection, (c) several parallels (implants) in each animal and finally (d) a model that is technically easy to perform.

Methods: Bone xenografts with steel plates were implanted intramuscularly in rats. To the xenografts, different inocula of Staphylococcus aureus and two strains of Staphylococcus epidermidis were added. The animals were observed for different time periods before the removal of the xenografts. The xenografts and steel plates were subjected to quantitative bacterial culture after sonication. Additional steel plates were subjected to scanning electron microscopy (SEM) for visualization of biofilm formation.

Results: Inoculation of bone grafts with S. aureus did produce a pyogenic infection in all animals. A chronic infection was established in rats where the bone grafts were inoculated with $S$. epidermidis. A bacterial inoculum of 100 colony-forming units (CFU) of S. epidermidis was adequate as a minimum infective dose. During a period of up until 42 days, the animals infected with S. epidermidis had no general or local signs of infection. According to the results of the quantitative bacterial culture of sonicate fluid and SEM, a biofilm was developed on all implants.

Conclusion: In the present in vivo model, a very small bacterial inoculum succeeded in establishing a chronic musculoskeletal implant infection where a biofilm was formed on the implants. The experimental model is easy to perform and allows several implants in each animal. The model could be useful for the study of biofilm formation in vivo on different implants and different surfaces.
\end{abstract}

Keywords: Experimental, Model, Infection, Chronic, Musculoskeletal

\section{Background}

A chronic infection is one of the most devastating complications to prosthetic joint surgery. The implant has to be removed, and the final functional result is often much inferior to what the patient would else have expected. These infections are considered to represent biofilm infections [1-3]. Most of the present knowledge of biofilm infections is based on in vitro studies [4]. However, the results of in vitro biofilm studies are not readily reproduced in vivo [5-7], and in vitro biofilm models are not models for the chronic in vivo infection [4]. During the last decades, several experimental models have been used to study the infected orthopaedic implant per se and biofilm formation on the implant in particular [8-22].

\footnotetext{
* Correspondence: eivind.witso@stolav.no

${ }^{1}$ Department of Orthopaedic Surgery, St Olav's University Hospital,

Trondheim, Norway

Full list of author information is available at the end of the article
}

In most of the abovementioned models, the foreign body is introduced into or on the tibia or the femur in mouse, rats and rabbits, and there is rarely more than one implant per animal $[8-14,16,18,19,21]$. Hence, a large number of animals are needed to achieve many parallels in each experimental group, necessarily implying housing and economic challenges. In some studies, precolonized implants are applied, i.e. the implants are colonized with bacteria in vitro $[9,11,12,19,21]$. In a rabbit femur model, Sheehan et al. [14] observed that 10 times more bacteria adhered to the implant when the bacteria were inoculated during the implantation of stainless steel and titanium wires compared to implants precolonized with bacteria.

Many models utilize large bacterial inocula [10, 17], and in some models, the rate of infection of the implants is between 30 and $100 \%$ [8, 10, 12, 14]. Finally, many of 
the models are technically rather demanding and barely possible to perform without being a surgeon.

The purpose of the present work was to establish an experimental model that mimics a chronic orthopaedic implant infection. The experimental model should have the following characteristics: (a) The surgical procedure should be technically easy to perform and allow the use of many parallels in relatively few animals. (b) A small bacterial inoculum should result in a chronic infection on all implants. Finally, (c) the infection established should have the characteristics of a chronic orthopaedic infection with no local or general signs of a purulent infection.

\section{Methods}

\section{An overview of the design of the study}

The Norwegian Council for Animal Experimentation approved the study (FOTS nr. 3854, 3987 and 4402). Rats were operated with intramuscular implantation of two bone grafts each containing two steel plates (Fig. 1). The bone grafts were seeded with strains of either Staphylococcus aureus (American Type Culture Collection (ATCC) 25923), S. epidermidis (ATCC 35984) and S. epidermidis (ATCC 12228) [21-23].

The extent of infection was evaluated by clinical observation of the animals and post mortem culture of muscle and spleen biopsies and quantitative culture of sonicated bone grafts. The degree of biofilm formation on steel plates was evaluated by sonication of the steel plates, followed by quantitative culture of the sonicate fluid and scanning electron microscopy (SEM) of the plates.

Three experiments were conducted:
1. Ten rats were operated with five rats in each experimental group. The bone grafts were inoculated with a $10-\mu$ l suspension containing $10^{4}$ colony-forming units (CFU) of S. aureus (ATCC 25923) and S. epidermidis (ATCC 35984), respectively. After 20 days, the bone grafts with their content were removed and further processed. Biopsies from the spleen and muscle surrounding the implants were collected for culture.

2. Twenty rats were operated, 10 rats in each experimental group. The bone grafts were inoculated with a $10-\mu$ l suspension of $S$. aureus (ATCC 25923) and S. epidermidis (ATCC 35984), respectively. In each experimental group, the bone grafts were contaminated with $10^{4}, 10^{3}$ and $10^{2}$ CFU in each subgroup of three rats, respectively. After 42 days, the bone grafts with their content were removed and further processed. Biopsies from the muscle were collected for culture.

3. Ten rats were operated, five rats in each experimental group. The bone grafts were inoculated with a $10-\mu$ l suspension of $10^{2} \mathrm{CFU}$ of $S$. epidermidis (ATCC 35984) and S. epidermidis (ATCC 12228), respectively. After 26 days, the bone grafts were removed and further processed. Biopsies from the muscle were collected for culture.

In each experimental group, one rat acted as a negative in vivo control.

\section{Bacterial strains}

Overnight cultures of S. aureus (ATCC 25923) and S. epidermidis (ATCC 35984 and ATCC 12228) were dissolved

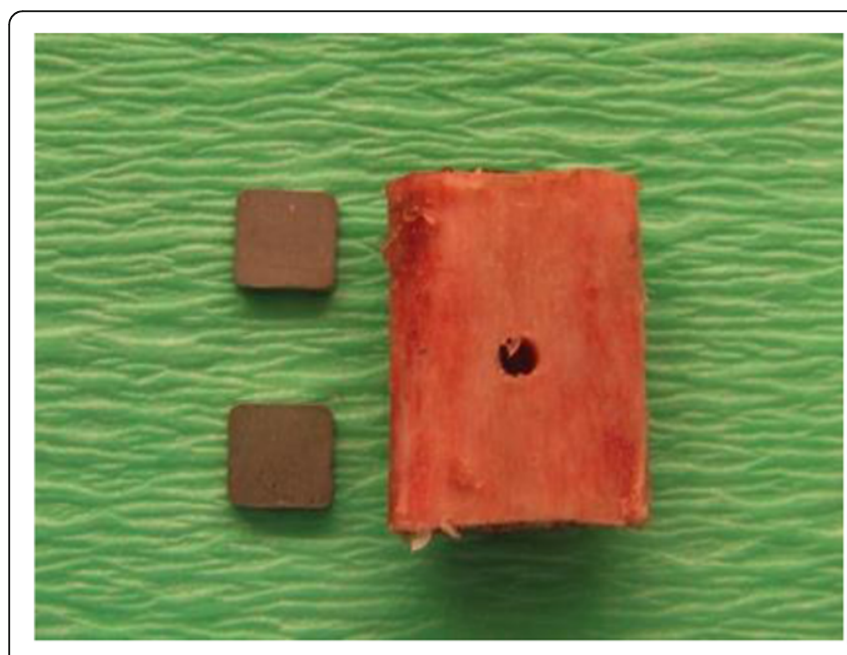

a

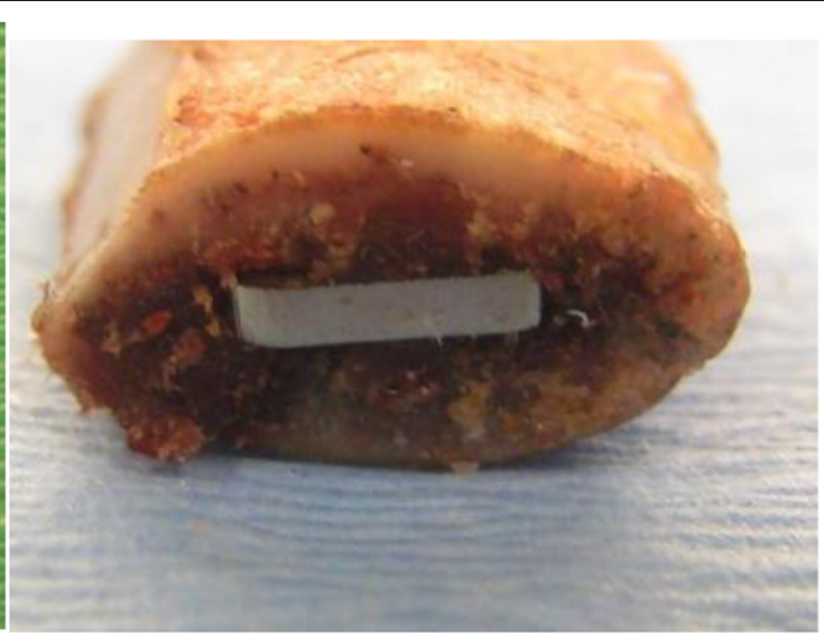

b

Fig. 1 a, b Xenograft and implants used for establishing a chronic infection model. Steel plates were impacted in both ends of a corticospongious xenograft (lamb costae) 
in 5\% glucose broth and incubated in order to obtain logphase growth. Ten-microliter aliquots of bacterial solution were serially diluted and seeded at regular time intervals after spectrometrical measurements enabling a standard curve for bacterial concentrations. This procedure allowed us to make the following 10- $\mu \mathrm{l}$ inoculate applied in the respective experiments: $10^{4}, 10^{3}$ and $10^{2} \mathrm{CFU}$ of ATCC 25923 and ATCC 35984 and $10^{2}$ CFU of ATCC 12228.

The respective bacterial solutions were kept on ice and brought to the Department of Comparative Medicine Core facility. Samples in duplicate from the bacterial solutions were seeded on blood agar plate at regular intervals during the operations to confirm the inoculum employed.

All seeded agars were incubated overnight at $37^{\circ} \mathrm{C}$ and enumerated in by using aCOLyte Colony Counter (Synbiosis, Cambridge, UK). In all experiments, representative colonies of bacteria harvested from infected animals were processed for pulsed-field gel electrophoresis using BIO-RAD CHEF MAPPER $^{\text {TM }}$ XA and compared with ATCC 25923, ATCC 35984 and ATCC 12228, respectively.

\section{The implant}

One hundred sixty steel plates (AISI 316-L, Scandinavian Customized Prosthesis $\left.{ }^{\circ}\right), 5 \times 5 \times 1 \mathrm{~mm}$, Ra $0.20(0.12-$ 0.31) $\mu \mathrm{m}$, were sonicated $\left(40 \mathrm{kHz}, 200 \mathrm{~W}\right.$, BactoSonic ${ }^{\circ}$, Bandelin $\mathrm{GmbH}$, Berlin) in sterile water for $2 \times 15 \mathrm{~min}$ before sterilization by autoclaving.

\section{The bone grafts}

Lamb costa from Norwegian white sheep was obtained from the slaughterhouse (Nortura Malvik', 7550 Hommelvik, Norway). The lambs had not been given any medication before they were killed by electricity. From the mid part of costae, 80 pieces of corticocancellous bone grafts were cut manually with a saw. With an electric sanding machine, the periost was removed and sharp corners were rounded. The grafts were 15.3 (14.5-16.5) $\mathrm{mm}$ in length and 11.9 (10.513.0) $\mathrm{mm}$ in breadth, and they weighted $1.16(1.00-1.27) \mathrm{g}$. A groove was made with a surgical knife in each end of the bone graft before a steel plate was gently inserted into each end of the graft with a forceps until the outer end of the steel plates were even with the cortical bone. At the mid part of the graft, a small hole was made into the bone marrow with a 1.9-mm drill (Fig. 1). The bone graft with the implant was then sterilized.

\section{The operation}

All operations were performed in a laminar air flow cabinet under standard operation theatre conditions. Forty male albino, outbred Wistar rats (HanTac:WH, Taconic, Denmark), 13 weeks old [12-14] and 360 (312-413) g, were operated. In all experiments, the operation field was shaved and washed with $70 \%$ ethanol. The animals were placed on sterile drapes, and their bodies were covered with sterile sheets, with a hole exposing the operation field. In all operations, the rats were given general sedation, and if necessary, buprenorphin as postoperative pain treatment. At the end of the experiment, the rats were killed with an intracardial injection of pentobarbital.

Briefly, a 4-cm-long incision was made in the midline at the proximal part of the back, and a subfascial/intramuscular cavity was made with a scissor on each side of the midline (right $=\mathrm{A}$, left $=\mathrm{B}$ ). Ten microliters of the respective bacteria solution was injected into the hole on the mid part of the bone graft $3 \mathrm{~s}$ before the bone grafts with the steel plates were implanted in each subfascial/ intramuscular cavity. In animals that acted as negative in vivo controls, the bone grafts were implanted directly into the cavities without any bacterial seeding. The skin was closed with a continuous locking madras suture. All animals were operated separately, with new dressing and instruments for each animal. During the first $24 \mathrm{~h}$ postoperatively, the animals were kept in separate cages and thereafter three and three in each cage. The animals were housed in a 12-h-night-daycycle environment. They were allowed free movement, standard food (rat and mouse diet "BKOO1E) and water ad libitum. The animals were weighed on the day of surgery and at weekly intervals throughout the study period. They were inspected daily for any complications, in particular signs of purulent wound secretion.

At the end of the experiment, a $5 \times 5 \times 5$-mm biopsy was first taken from the spleen by a laparotomy (experiment I). The old scar at the back of the animal was then opened, and any possible signs of local infection were noted. Two $5 \times 5 \times 5 \mathrm{~mm}$ biopsies were harvested from the muscle surrounding the bone grafts. The bone grafts were then removed, first graft A and then graft B. By applying a gentle pressure by forceps over the mid part of the graft, the graft would split longitudinally and then the graft could be opened like a book. One of the two steel plates from bone graft A was immediately put in fixation solution for SEM. Next, the bone grafts with its content were separately put into sterile plastic containers and were immediately placed on ice for further processing.

The duration of the two surgical procedures in each animal was approximately 10 and $7 \mathrm{~min}$, respectively.

\section{Postoperative processing of spleen biopsies, muscle biopsies, bone grafts and steel plates}

All specimens were kept on ice and brought to the laboratory for immediately processing.

The spleen biopsies were grounded in a mortar with 1 $\mathrm{ml}$ of sterile saline, after which $50 \mu \mathrm{l}$ of the suspension was seeded onto blood agar plate. The muscle biopsies were grounded with $3 \mathrm{ml}$ of sterile saline, the suspension was serially diluted and $10 \mu \mathrm{l}$ was seeded onto blood agar 
plate. While still on ice, each steel plate was removed from the bone grafts with a pincer. In a sterile plastic tube with $3 \mathrm{ml}$ saline, each steel plate was gently stirred $(600 \mathrm{rpm})$ for $10 \mathrm{~s}$. This washing procedure was repeated twice (three times in experiment II), and the steel plate was transferred each time with a new sterile pincer. After the last washing procedure, the plate was transferred to a sterile glass tube with $3 \mathrm{ml}$ sterile saline for removal of biofilm [24]. Briefly, the glass tube with its content was vortex mixed at $2400 \mathrm{rpm}$ for $30 \mathrm{~s}$, followed by sonication $\left(40 \mathrm{kHz}, 200 \mathrm{~W}\right.$, BactoSonic ${ }^{\circ}$, Bandelin $\mathrm{GmbH}$, Berlin) for $5 \mathrm{~min}$, and then a second vortex mixing at $2400 \mathrm{rpm}$ for $30 \mathrm{~s}$. Ten microliters of the sonicate fluid was serially diluted and seeded onto blood agar plate. After completion of sonication, the second steel plate from bone graft A was put into a fixation solution for SEM. Finally, the bone grafts were vortex mixed and sonicated in $6 \mathrm{ml}$ of sterile saline according to the same procedure as the steel plates (preliminary studies had shown that this procedure yielded an equivalent amount of bacteria as if the bone grafts had been grounded in a mortar). From this sonicate fluid, $10 \mu \mathrm{l}$ was serially diluted and seeded onto a blood agar plate.

From the three animals that acted as negative in vivo control, one plate from each of the bone grafts was processed directly for SEM, while the other plates were sonicated. After sonication, aliquots of sonicate fluid were seeded onto a blood agar plate. Sonicated plates from negative in vivo control animals were processed for SEM.

\section{Scanning electron microscopy (SEM) of steel plates}

For SEM, the steel plates were briefly rinsed with $0.1 \mathrm{M}$ Hepes buffer (Sigma-Aldrich) at pH 7.2, then fixed in 2.5\% glutaraldehyde (Chemi-Teknik AS), 2\% paraformaldehyde (VWR, Part of Avantor) and 0.075\% Ruthenium Red (Sigma-Aldrich) in $0.1 \mathrm{M}$ Hepes buffer for $4 \mathrm{~h}$ at room temperature. Afterwards, the specimens were rinsed in $0.1 \mathrm{M}$ Hepes buffer and then dehydrated through a graded series of ethanol (Antibac AS), (10\%, 25\%, 50\%, 70\%), and 90\%] for $7 \mathrm{~min}$ each and then 3 times in 100\% ethanol for 10 min each. After dehydration, the specimens were dried using a chemical hexamethyldisilazane (HMDS) (SigmaAldrich), first exchange with 50\% solution of HMDS diluted with $100 \%$ ethanol for $20 \mathrm{~min}$, then three exchanges with 100\% HMDS for 20 min each. Finally, HMDS were removed and the specimens were allowed to air-dry in a fume hood overnight. Dried samples were mounted on stubs using double-sided conductive adhesive carbon tape (both from Chemi-Teknik AS). Specimens were then coated with a 30-nm-thick layer of gold/palladium (Au/ Pd) using a sputter coater (polaron) $(2.5 \mathrm{kV}, 20 \mathrm{~mA}, 3$ $\mathrm{min}$ ). Specimens were examined with a JSM 6480 (JEOL) scanning electron microscope with digital imaging capabilities. The secondary electron images were collected at an acceleration voltage of $20 \mathrm{kV}$ and digitalized as TIFF computer files.

\section{Presentation of data}

Data are presented as either mean (total range) or mean \pm 1 standard deviation $(\mathrm{SD})$.

\section{Results}

Animals infected with S. aureus (ATCC 25923)

In brief, inoculating the bone grafts with $S$. aureus resulted in an acute postoperative infection, and none of the animals $(n=13)$ infected with $S$. aureus developed a chronic infection. All animals had macroscopic signs of a purulent infection in the subcutaneous and muscle tissue. In experiment I, the total number of CFU in the eight bone grafts contaminated with $S$. aureus was $46( \pm 32) \cdot 10^{7}$. One steel plate was lost during processing, leaving 11 steel plates for sonication. The CFU/millilitre in sonicate fluid was $65( \pm 40) \cdot 10^{4}$. Plates prepared for SEM showed bacterial colonization on all plates.

None of the rats infected with $S$. aureus fulfilled experiment II: after 24 days, $4 / 9$ rats had severe purulent wound infections and the animals were sacrificed. In the other $5 / 9$ rats, the bone grafts had already been spontaneously expelled.

\section{Animals infected with S. epidermidis (ATCC 35984 and ATCC 12228)}

Of the 25 animals, one rat died postoperatively due to respiratory depression. In one animal, the bone grafts and the implants were infected with a $S$. aureus contaminant (different from ATCC 25923). In the rest of the infected animals $(n=19)$, the causative microbe was identified as ATCC 35984 and ATCC 12228, respectively. All animals, including controls $(n=23)$, were healthy and gained weight, and cultures of biopsies from the spleen were negative.

In the 19 infected animals, there were no macroscopic signs of infection of the skin in the subcutaneous or subfascial tissue. All bone grafts remained positioned intramuscularly. In five animals, the bone grafts were surrounded by a pseudocapsule filled with sanguinolent fluid. ATCC 35984 and ATCC 12228 were cultured in respective biopsies taken from the muscle adjacent to the contaminated bone grafts. The total number of CFU in bone grafts at the right side of the animal (graft A) and the left side (graft B) was similar (data not shown). Irrespective of the bacterial inoculum, the length of the infectious period and the bacterial species (S. epidermidis ATCC 35984 and S. epidermidis ATCC 12228), the total number of CFU in bone grafts were about $10^{7}$ and bacterial growth in sonication fluid was about $10^{3} \mathrm{CFU} / \mathrm{ml}$ (Tables 1 and 2). Examination with SEM showed bacterial growth, individually and in colonies, on all plates contaminated with S. epidermidis (Fig. 2a-c). 
Table 1 Steel implants infected with S. epidermidis ATCC 35984 in an in vivo rat model

\begin{tabular}{lllll}
\hline Inoculum (CFU) & $0.7(0.7-0.8) 10^{4}$ & $1.1(1.1-1.1) 10^{4}$ & $0.9(0.7-1.1) 10^{3}$ & $1.4(1.3-1.4) 10^{2}$ \\
\hline Time period (days) & 20 & 42 & 42 & 42 \\
Rats $(n)$ & 4 & $2^{a}$ & 3 & $2^{a}$ \\
Bone grafts $(n)$ & 8 & 4 & 6 & 4 \\
Steel plates $(n)^{\text {b }}$ & 12 & 6 & 9 & 6 \\
Bacterial growth in bone grafts (total CFU) & $110( \pm 50) \cdot 10^{5}$ & $69( \pm 41) \cdot 10^{5}$ & $96( \pm 65) \cdot 10^{5}$ & $170( \pm 36) \cdot 10^{5}$ \\
Bacterial growth in sonicate fluid (CFU/ml) & $217( \pm 122) \cdot 10^{2}$ & $31( \pm 22) \cdot 10^{2}$ & $68( \pm 112) 10^{2}$ & $32( \pm 21) 10^{2}$ \\
\hline
\end{tabular}

The total number of CFU of S. epidermidis ATCC 35984 are listed as a result of different time periods for infection and different inoculum size (experiments I and II). The number of CFU recovered from bone grafts and sonicate fluid are presented as mean ( \pm 1 SD).

${ }^{a}$ One rat died postoperatively. In one rat, the bone grafts were contaminated

${ }^{\mathrm{b}}$ From each animal, one steel plate was processed for SEM and three steel plates were sonicated

In control animals, no bacterial growth was recorded in the spleen tissue, muscle tissue, bone graft or sonicate fluid. No bacteria were observed on steel plates prepared for SEM.

\section{Discussion}

We here present a new experimental model for the study of chronic implant infections in the musculoskeletal system. We found that a bone xenograft with implants contaminated with a very low inoculum of $S$. epidermidis in this in vivo model resulted in a chronic implant infection.

Numerous models for in vivo studies of skeletal staphylococci implant infections have been presented during the last 50 years $[22,25]$. In vivo studies are justified due to the interaction between the host, the bacteria and the implant, which cannot be studied in vitro [26]. Furthermore, clinical trials on osteomyelitis are difficult to conduct due to a large variability of the disease, a low incidence in most countries, a heterogeneous population and a broad range of causative microbes [22, 25].

The strength of the present study is that a very small inoculum (100 CFU) of S. epidermidis was capable of establishing a chronic infection, and a biofilm was formed on all implants. This is probably more comparable to the clinical situation of preoperative contamination of devices as opposed to the massive infectious doses often employed in many experimental in vivo models. Even after 6 weeks, none of the animals infected with $S$. epidermidis had general or local signs of an infection, and the animals had a normal gain in body weight. The model allowed several implants (parallels) in each animal, thus reducing the number of experimental animals needed. In the present study, a total of two xenografts and four implants were studied in each animal, but the number of grafts (implants) could easily have been doubled. The surgical technique applied in the present model is easy to perform. After training and supervision, operation of the animals can be performed by personnel not necessarily possessing complete surgical skills. Finally, in the present model, the risk of bacterial contamination from the fur and skin of the animal was negligible. Sonication of bone grafts and steel plates in the present study was performed according to standard procedure [27]. Bacterial growth in sonicate fluid has been considered a proof of the presence of biofilm in both in vitro and clinical studies [27-30]. In the present study, SEM was employed to visualize the presence of bacterial adherence on steel plates. This is a qualitative evaluation which has been used in both in vitro and clinical studies [31, 32]. In a pilot study, we tried to quantify the number of bacterial colonies (i.e. more than five bacteria in a cluster). At a magnification of $\times 2000$ we scanned about $7 \%$ of the total area of the plate. This method allowed us to quantify the total number of bacterial colonies in plates subjected to the same experimental setup, and we found that parallels had a similar number of bacterial colonies (data not shown). This procedure was extremely time-consuming. But at

Table 2 Recovery of bacteria from rats inoculated with a small inoculum of two S. epidermidis strains

\begin{tabular}{lll}
\hline & ATCC 35984 & ATCC 12228 \\
\hline Inoculum (CFU) & $1.02(0.80-1.12) \cdot 10^{2}$ & $0.92(0.87-1.04) \cdot 10^{2}$ \\
Rats $(n)$ & 4 & 4 \\
Bone grafts $(n)$ & 8 & 8 \\
Steel plates $(n)^{\text {a }}$ & 12 & 12 \\
Bacterial growth in bone grafts (total CFU) & $341( \pm 266) \cdot 10^{5}$ & $109( \pm 79) \cdot 10^{5}$ \\
Bacterial growth in sonicate fluid (CFU/ml) & $159( \pm 100) \cdot 10^{2}$ & $113( \pm 94) \cdot 10^{2}$ \\
\hline
\end{tabular}

${ }^{a}$ From each animal, one steel plate was processed for SEM and three steel plates were sonicated

The number of CFU in bone grafts and sonicate fluid are presented as mean ( \pm 1 SD) 


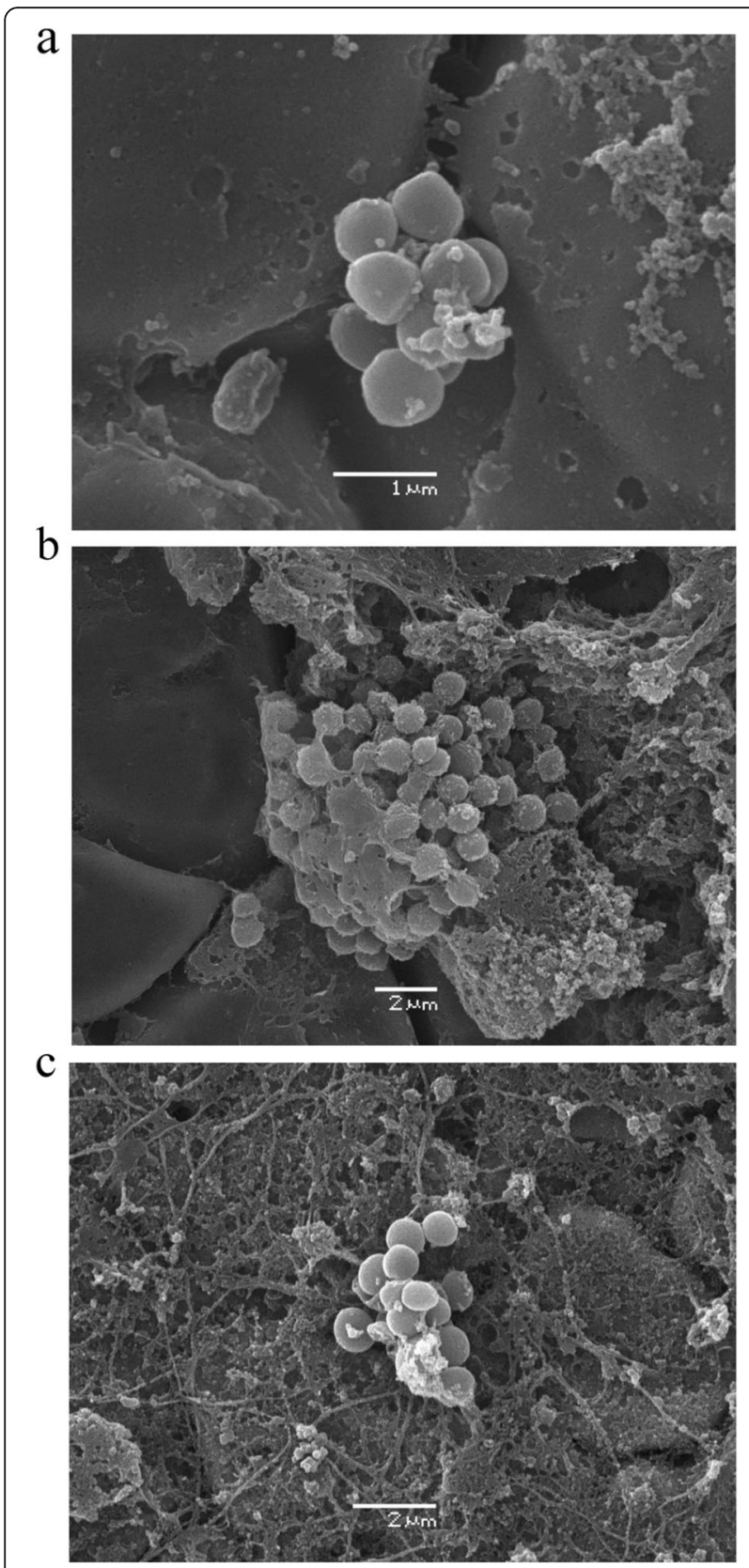

Fig. 2 a-c Scanning electron micrograph showing bacterial growth with biofilm in small colonies on steel plates (S. epidermidis, ATCC 35984)

least it was possible to quantify the number of bacterial colonies on the plate, as opposed to what would have been feasible on a curved surface, as for example the surface of pins. In the present study, neither bacterial culture of sonicate fluid nor SEM was employed quantitatively, but as a means to study if even an extremely small bacterial inoculum could create a chronic biofilm infection.

The finding of a low-virulent bacterium as S. epidermidis resulting in a chronic infection is in accordance with clinical observations [33]. In the present study, we used a biofilm- forming S. epidermidis (ATCC 35984) and a non-biofilmforming S. epidermidis (ATCC 12228) [23, 34]. As observed in other studies, the differences in biofilm-forming abilities were not reproduced in vivo [6, 7]. To our best knowledge, this observation has not been further addressed in specific experimental models of orthopaedic implant infections.

A weakness of the present study includes that we did not manage to create a chronic infection when the xenografts were contaminated with $S$. aureus. Other authors have succeeded in creating a chronic infection in rat femora when a foreign body introduced intramedullary (pin or wire) was contaminated with S. aureus [22]. In our model, $S$. aureus resulted in an acute suppurative infection, probably due to the avascular status of the xenograft and the virulence of the organism. We do not know to what degree the use of a xenograft will influence on the applicability of the model. However, the present model is first and foremost a model for studying implant infections, and not a model for studying osteomyelitis.

The limitations of the present study were that only one bacterial species (i.e. staphylococci) and one type of implant (i.e. steel) were studied. Inoculation with other low-virulent bacteria, as for example Cutibacterium acnes, would possibly also result in a chronic infection, but this has yet to be shown. We could have used other imaging techniques, both to make a qualitative and quantitative evaluation of the presence of a biofilm. Confocal laser scanning microscopy has been employed to study the biofilm per se in vivo [21, 31]. A quantitative evaluation of biofilm formation has been studied with different fluorescence techniques and with different degree of success [21, 35]. Jørgensen et al. [21] employed a mouse model where precolonized pins were inserted into mouse tibia. Bioluminescent $S$. aureus allowed bioluminescent imaging. However, bioluminescence activity decreased during the study period although the bacterial load was the same. This phenomenon was explained by precolonization of the implants and emphasizes that culture of sonication fluid was the most reliable quantitative method. Finally, the results observed in this model do only relate to rats. Rats are known to have a strong immune system [22], and other results could have been observed if for example rabbits had been used.

\section{Conclusions}

The present model is primarily a model for the study of biofilm formation on implants in vivo. A very small and clinically relevant bacterial inoculum creates a low virulent chronic implant infection with no macroscopic sign of infection. The model could be employed when the effect of local antibiotics or surface modification of the implant is the subject of interest. The present in vivo model could possibly also be used to study the effect of per oral or parenteral antibiotic treatment in cases of musculoskeletal and foreign body infections, but this has to be explored. 


\section{Abbreviations}

ATCC: American Type Culture Collection; CFU: Colony-forming units; SEM: Scanning electron microscopy

\section{Acknowledgements}

Parts of this study were performed at the Cellular and Molecular Imaging Core Facility (CMIC), Norwegian University of Science and Technology (NTNU). CMIC is funded by the Faculty of Medicine at NTNU and Central Norway Regional Health Authority.

\section{Authors' contributions}

EW was responsible for the study design, most of the laboratory work and writing the article. LH was responsible for SEM and revision of the manuscript. KL assisted in most of the laboratory work and in the revision of the manuscript. KB was involved in the design of the study and assisted in the revision of the manuscript. All authors read and approved the final manuscript.

\section{Funding}

No funding was obtained for the current study.

\section{Availability of data and materials}

All data generated and analyzed during this study are included in this published article.

\section{Ethics approval and consent to participate}

The study was approved by The Norwegian Council for Animal Experimentation.

\section{Consent for publication}

Not applicable

\section{Competing interests}

The authors declare they have no competing interests.

\section{Author details}

'Department of Orthopaedic Surgery, St Olav's University Hospital, Trondheim, Norway. ${ }^{2}$ Department of Clinical and Molecular Medicine, Norwegian University of Science and Technology, Trondheim, Norway. ${ }^{3}$ Department of Medical Microbiology, St Olav's University Hospital, Trondheim, Norway.

Received: 17 June 2019 Accepted: 5 January 2020

Published online: 21 January 2020

\section{References}

1. Costerton JW, Geesey GG, Cheng KJ. How bacteria stick. Sci Am. 1978;238(1): 86-95.

2. Gristina AG. Biofilm-centered infection: microbial adhesion versus tissue integration. Science. 1987;237(4822):1588-95.

3. Zimmerli W, Moser C. Pathogenesis and treatment concepts of orthopaedic biofilm infections. FEMS Immunol Med Microbiol. 2012;65(2):158-68.

4. Bjarnsholt $T$, Alhede M, Alhede M, Eickhardt-Sørensen SR, Moser C, Kühl M, Jensen $P \varnothing$, Høiby N. The in vivo biofilm. Trends Microbiol. 2013; 21(9):466-74.

5. Vandecasteele SJ, Peetermans WE, Merckx R, Van Eldere J. Expression of biofilm-associated genes in Staphylococcus epidermidis during in vitro and in vivo body infection. J Infect Dis. 2003:188(5):730-7.

6. Francois P, Quoc PHT, Bisognano C, Kelley WL, Lew DP, Schrenzel J, Cramton SE, Götz F, Vaudaux P. Lack of biofilm contribution to bacterial colonisation in an experimental model of foreign body infection by Staphylococcus aureua and Staphylococcus epidermidids. FEMS Immunol Med Microbiol. 2003:23:135-40.

7. Chokr A, Leterme D, Watier D, Jabbouri S. Neither the presence of ica locus, nor in vitro-biofilm formation ability is a crucial parameter for some Staphylococcus epidermidids strains to maintain an infection in a guinea pig tissue cage model. Microb Pathog. 2007;42:94-7.

8. Arens S, Schlegel U, Printzen G, Ziegler WJ, Perren SM, Hansis M. Influence of materials for fixation implants on local infection. J Bone Joint Surg Br. 1996;78(4):647-51.
9. Gracia E, Laclériga A, Monzón M, Leiva J, Oteiza C, Amorena B. Application of a rat osteomyelitis model to compare in vivo and in vitro the antibiotic efficacy against bacteria with high capacity to form biofilms. J Surg Res. 1998:79:146-53.

10. Arens S, Eijer H, Schlegel U, Printzen G, Perren SM, Hansis M. Influence of the design for fixation implants on local infection: experimental study of dynamic compression plates versus point contact fixators in rabbits. $J$ Orthop Trauma. 1999;13(7):470-6.

11. Monzón M, Gracía-Álvarez F, Laclériga A, Gracia E, Leiva J, Oteiza C, Amorena B. A simple infection model using pre-colonized implants tp reproduce rat chronic Staphylococcus aureus osteomyelitis and study antibiotic treatment. J Orthop Res. 2001;19:820-6.

12. Monzón M, Gracía-Álvarez F, Laclériga A, Amorena B. Evaluation of four experimental osteomyelitis infection models by using precolonized implants and bacterial suspensions. Acta Orthop Scand. 2002;73(1):11-9.

13. Lucke M, Schmidmaier G, Sadoni S, Wildmann B, Schiller R, Haas NP, Raschke M. Gentamicin coating of metallic implants reduces implant-related osteomyelitis in rats. Bone. 2003;32:521-31.

14. Sheehan E, McKenna J, Mulhall KJ, Marks P, McCormack D. Adhesion of Staphylococcus to orthopaedic metals, an in vivo study. J Orthop Res. 2003; 22:39-43.

15. Craig MR, Poelstra KA, Sherrell JC, Kwon MS, Belzile EL, Brown TE. A novel total knee arthroplasty model in rabbits. J Orthop Res. 2005;23:1100-4.

16. Lucke M, Wildmann B, Sadoni S, Surke C, Schiller R, Stemberger A, Raschke M, Haas NP, Schmidmaier G. Systemic versus local application of gentamicin in prophylaxis of implant-related osteomyelitis in a rat model. Bone. 2005; 36:770-8.

17. Alt V, Bitschnau A, Österling J, Sewing A, Meyer C, Kraus R, Meissner SA, Wenisch S, Domann E, Schnettler R. The effects of combined gentamicinhydroxyapatite coating for cementless joint prostheses on the reduction of infection rates in a rabbit infection prophylaxis model. Biomaterials. 2006; 27(26):4627-34.

18. Darouiche RO, Mansouri MD, Zakarevicz D, Alsharif A, Landon GC. In vivo efficacy of antimicrobial-coated devices. J Bone Joint Surg Am. 2007;89:792-7.

19. Li D, Gromov K, Søballe K, Puzas JE, O'Keefe RJ, Awad H, Drissi H, Schwarz EM. Quantitative mouse model of implant-associated osteomyelitis and the kinetics of microbial growth, osteolysis, and humoral immunity. J Orthop Res. 2008:26(1):96-105.

20. Bernthal N, Stavrakis Al, Billi F, Cho JS, Kremen TJ, Simon SI, Cheung AL, Finerman GA, Lieberman JR, Adams JS, Miller LS. A mouse model of postarthroplasty Staphylococcus aureus joint infection to evaluate in vivo efficacy of antimicrobial implant coatings. PLoS One. 2010;5(9):e12580.

21. Jørgensen NP, Meyer R, Dagnæs-Hansen F, Fuursted K, Petersen E. A modified chronic infection model for testing treatment of Staphylococcus aureua biofilms on implants. PLoS One. 2014:9(10):e103688.

22. Reiner W, Hunter JG, O'Mallet NT, Southgate RD, Schwarz EM, Kates SL. A systematic review of animal models for Staphylococcus aureus osteomyelitis. Eur Cell Mater. 2015;27:196-212.

23. Zhang YQ, Ren SX, Li HL, Wang YX, Fu G, Yang J, Qin ZQ, Miao YG, Wang WY, Chen RS, Shen Y, Chen Z, Yuan ZH, Zhao GP, Qu D, Danchin A, Wen YM. Genome-based analysis of virulence genes in a nonbiofilm-forming Staphylococcus epidermidis strain (ATCC 12228). Mol Microbiol. 2003;49(6):1577-93.

24. Kobayashi H, Oethinger M, Tuohy MJ, Procop GW, Bauer TW. Improved detection of biofilm-formative bacteria by vortexing and sonication: a pilot study. Clin Orthop Relat Res. 2009:467(5):1360-4.

25. Norden CW. Lessons learned from animal models of osteomyelitis. Rev Infect Dis. 1988;10:103-10.

26. Handke JD, Rupp ME. In vivo models of biomaterial-associated infection by biofilm-forming staphylococci. In: Pace JL, Rupp ME, Finch RG, editors. Biofilm, infection and antimicrobial therapy. Boca Raton: Taylor \& Francis Group; 2006. p. 289-303.

27. Bjerkan G, Witsø E, Bergh K. Sonication is superior to scraping for retrieval of bacteria in biofilm on titanium and steel surfaces in vitro. Acta Orthop. 2009:80:245-50.

28. Trampuz A, Piper KE, Jacobson MJ, Hanssen AD, Unni KK, Osmon DR, Mandrekar JN, Cockerill FR, Steckelberg JM, Greenleaf JM, Patel R. Sonication of removed hip and knee prostheses for diagnosis of infection. NEJM. 2007; 357:654-63.

29. Portillo ME, Salvado M, Alier A, Martinez S, Sorli L, Horcajada JP, Puig L. Advantages of sonication fluid culture for the diagnosis of prosthetic joint infection. J Inf Secur. 2014;69:35-41. 
30. Bjerkan G, Witsø E, Nor A, Viset T, Løseth K, Lydersen S, Persen L, Bergh K. A comprehensive microbiological evaluation of fifty-four patients undergoing revision surgery due to prosthetic joint loosening. J Med Microbiol. 2012;61:572-81.

31. Gorman SP, Adair CG, Mawhinney WM. Incidence and nature of peritoneal catheter biofilm determined by electron and confocal laser scanning microscopy. Epidemiol Infect. 1994;112:551-9.

32. Arnold JW, Bailey GW. Surface finishnes on stainless steel reduce bacterial attachment and early biofilm formation: scanning electron and atomic force microscopy study. Poult Sci. 2000;79:1839-45.

33. Lutro O, Langvatn H, Dale H, Schrama JC, Hallan G, Espehaug B, Sjursen H, Engesaeter LB. Increasing resistance of coagulase-negative staphylococci in total hip arthroplasty infections: 278 THA-revisions due to infection reported to the Norwegian Arthroplasty Register from 1993 to 2007. Adv Orthopaed. 2014:2014:580359

34. Williams DL, Bloebaum RD. Observing the biofilm matrix of Staphylococcus epidermidis ATCC 35984 grown using the CDC biofilm reactor. MicrosC Microanal. 2010;16(2):143-52.

35. Kajiyama S, Tsurumoto T, Osaki M, Yanagihara K, Shindo H. Quantitative analysis of Staphylococcus epidermidis biofilm on the surface of biomaterial. J Orthop Sci. 2009;14:769-75.

\section{Publisher's Note}

Springer Nature remains neutral with regard to jurisdictional claims in published maps and institutional affiliations.

Ready to submit your research? Choose BMC and benefit from:

- fast, convenient online submission

- thorough peer review by experienced researchers in your field

- rapid publication on acceptance

- support for research data, including large and complex data types

- gold Open Access which fosters wider collaboration and increased citations

- maximum visibility for your research: over $100 \mathrm{M}$ website views per year

At BMC, research is always in progress.

Learn more biomedcentral.com/submissions 